\title{
Empreendedorismo na agricultura extensiva: análise da literatura internacional
}

\section{Entrepreneurship in extensive agriculture: analysis of international literature}

\author{
Luiz Diego Vidal Santos ${ }^{1 *}$, Pedro Vinícius Bertulino de Menezes², Francisco Sandro \\ Rodrigues Holanda ${ }^{2}$, Alexsandro da Fonseca de Sousa ${ }^{2}$, Renisson Neponuceno de Araújo \\ Filho $^{3}$, Catuxe Varjão de Santana Oliveira ${ }^{4}$, Adison Machado Enes ${ }^{5}$, Welington Gonzaga do \\ Vale $^{5}$
}

\begin{abstract}
RESUMO
Considerando que o tema empreendedorismo e agricultura extensiva ainda é pouco discutido em conjunto e que se mostra de suma importância o presente estudo teve por objetivo demonstrar quais os últimos avanços do empreendedorismo agrícola para o meio agrícola com atividade extensiva por meio de uma análise sistemática da literatura internacional. Como critério de categorização da importância e semelhança dos artigos estudados, foram utilizados os princípios da bibliometria e seguidas as recomendações do Manual de Avaliação do Sistema de Intervenção Cochrane. Os resultados apontaram os quatro temas mais comuns: o primeiro foi um artigo sobre empreendedorismo digital no contexto agrícola; a segunda parte foi composta por pesquisas relacionadas à educação para o empreendedorismo; a terceira parte trata dos tipos e características das empresas agrícolas, e a quarta parte envolve os aspectos psicológicos do empreendedorismo. A consideração sobre os aspectos económicos, como apoio financeiro, relações parentais, educação rural e apoio governamental para esses produtores, foram fundamentais para o desenvolvimento de novos negócios que tenham sucesso nas áreas rurais.
\end{abstract}

Palavras-chave: Agricultura familiar; Negócios sustentáveis; Revisão sistemática; Cochrane

\section{ABSTRACT}

Considering that the subject of entrepreneurship and extensive agriculture is still little discussed together and that it is of paramount importance, this study aimed to demonstrate the latest advances in agricultural entrepreneurship for the agricultural environment with extensive activity through a systematic analysis of the literature International. As a criterion for categorizing the importance and similarity of the articles studied, the principles of bibliometrics were used and the recommendations of the Cochrane Intervention System Assessment Manual were followed. The results pointed to the four most common themes: the first was an article on digital entrepreneurship in the agricultural context; the second part consisted of research

1 Universidade Federal de Sergipe-UFS/Programa de Pós-graduação em Ciências da Propriedade Intelectual-PPGPI. *E-mail: vidal.center@academico.ufs.br

${ }^{2}$ Universidade Federal de Sergipe-UFS/Departamento de Engenharia Agronômica-DEA

${ }^{3}$ Universidade Federal do Tocantins-UFT

${ }^{4}$ Instituto Federal de Sergipe-IFS

${ }^{5}$ Universidade Federal de Sergipe-UFS/Departamento de Engenharia Agrícola-DEAgri 
related to entrepreneurship education; the third part deals with the types and characteristics of agricultural enterprises, and the fourth part involves the psychological aspects of entrepreneurship. Consideration of economic aspects such as financial support, parental relationships, rural education, and government support for these producers were fundamental to the development of new businesses that are successful in rural areas.

Keywords: Family farming; Sustainable businesses; Systematic review; Cochrane

\section{INTRODUÇÃO}

A relevância da agricultura para o mundo é indiscutível, pois é através desta atividade que se fornecem alimentos e matérias-primas para diversos setores da economia. Mesmo diante dos desafios de superpopulação, mudanças climáticas, dificuldades na produção agrícola em grande escala e competição por recursos no mundo de hoje, essa atividade tem um papel único (Riegler, 2018; Rockstrom et al., 2017).

Neste setor econômico, a agricultura extensiva, especialmente a agricultura familiar, é parte essencial do ponto de vista econômico, social e ambiental, pois atende aos requisitos de sustentabilidade e elimina a pobreza no meio rural, auxiliando os agricultores na geração de renda, oportunidades de trabalho e produção competitiva com os grandes produtores, resultando comidas mais saudáveis resultado de lavouras mais diversificadas, nas quais os produtores rurais cultivam várias safras na mesma terra (Santana, 2014).

Métodos e inovações para construir o empreendedorismo permitem que todos os setores da economia e da sociedade superem os desafios diários (Cuéllar-Gálvez, Aranda Camacho e Mosquera-Vásquez, 2018). Portanto, a agricultura extensiva precisa fazer uso do empreendedorismo e inovação comercial como estratégia para desenvolver sua propriedade/produção, utilizando todos os recursos disponíveis a fim de melhorar e até mesmo criar produtos e serviços (Julien, 2017).

Atividades de tomada de decisão que usam novos métodos em áreas rurais frequentemente envolvem extensa pesquisa em uma grande quantidade de literatura. Tais atividades são trabalhosas, demoradas e difíceis de ser interpretadas pelos pesquisadores (Xiao et al., 2019). Nessa perspectiva, parece vantajoso desenvolver mecanismos seguros, automatizados, organizados e controláveis na coleta de evidências e considerar a validade e relevância das afirmações encontradas (Idri, Abnane e Abran, 2018), especialmente na escolha de evidências, sobre novos para produção agrícola e métodos de produção. Esse mecanismo deve facilitar aos usuários a otimização do tempo e do trabalho necessários para manter a confiabilidade dos resultados (Rockstrom et al., 2017). 
As revisões sistemáticas (RS) em documentos acadêmicos podem alcançar tais objetivos, de forma repetível e controlável já que, estes métodos mais estruturados de revisão procuram reunir evidências que se enquadrem em critérios de elegibilidade pré-especificados para responder a uma pergunta de pesquisa específica (Chandler et al., 2021), especulando sobre o estado da arte do tema de interesse (Keele, 2007; Medeiros et al., 2015).

Para identificar, avaliar e interpretar os resultados relevantes das revisões sistemáticas em trabalhos acadêmicos publicados, de acordo com os princípios das regras empíricas relacionadas aos levantamentos bibliométricos na literatura, atualmente são amplamente adotados e aceito os procedimentos de classificação, descrição e qualificação de documentos que adotam as leis (i) Lei da Produtividade do Pesquisador ou Lotka; (ii)

A lei da dispersão do conhecimento científico ou lei de Bradford e (iii) a lei da distribuição e frequência das palavras no texto ou lei de Zipf, elaborada em 1926, 1934 e 1949. Tais leis foram projetadas para mapear e compreender o domínio do conhecimento, principalmente considerando os dados gerados pela operação de publicação.

O método de análise do conteúdo trata-se de um conjunto de técnicas aplicadas para a análise de dados qualitativos, que se utilizam a ocorrência de palavras e similitudes dos termos concentrados em corpus de modo a determinar a importância destas, no contexto do assunto abordado (Cassettari et al., 2015). Métodos de Revisão sistemática aplicado às pesquisas sobre empreendedorismo na produção agrícola extensiva pode contribuir para a expansão do campo do conhecimento científico, levantamento de metas, ideias e o momento atual sobre o tema, por meio de conteúdos acadêmicos.

Considerando que o tema empreendedorismo e agricultura extensiva ainda é pouco discutido em conjunto e que se mostra de suma importância o presente estudo teve por objetivo demonstrar quais os últimos avanços do empreendedorismo agrícola para o meio agrícola com atividade extensiva por meio de uma análise sistemática da literatura internacional.

\section{MATERIAL E MÉTODOS}

Buscando contemplar o desenvolvimento das fases deste estudo, dois revisores examinaram independentemente os resultados dos dados obtidos em cada rodadas desta pesquisa, para isto foi adaptado as recomendações do Cochrane Handbook for Systematic Reviews of Interventions (Chandler et al., 2021), adotando como procedimentos deste estudo as sete rodadas: (i) Formulação da pergunta, (ii) Localização dos estudos (artigos), (iii) Seleção dos estudos, (iv) Extração dos estudos, (v) Análise e interpretação dos dados e (vi) Síntese da revisão e qualificação dos trabalhos, para isto, como modelo estrutural do trabalho, este trabalho 
adotou como diretrizes o método sistemático Cochrane Central Register of Controlled Trials (Bell et al., 2005) adaptando-o para esta pesquisa.

Para atender os parâmetros bibliométricos da pesquisa segundo a Lei de Lotka e de Bradford foram utilizados o método SCAS utilizando o software StArt (Hernandes et al., 2012), já R. Bibliometrix (Aria e Cuccurullo, 2017) foi utilizado para o desenvolvimento dos cálculos bibliométricos. já para a análise textual dos artigos selecionados foi utilizado o software Iramuteq (Salviati, 2017).

\section{Rodada 1: Formulação da pergunta}

Este estudo atribuiu o seguinte questionamento: Qual o estado da arte da literatura cientifica sobre o empreendedorismo e a relação deste na produção agrícola extensiva, e quais os últimos avanços do empreendedorismo agrícola para o meio agrícola com atividade extensiva.

\section{Rodada 2: Extração dos estudos}

$\mathrm{Na}$ segunda rodada foi realizada uma busca com descritores primários nas bases de dados Scopus (Elsevier) e Web of Science (Clarivate Analytics). Como estratégia para busca da produção acadêmica a ser utilizada nesta rotina, foram definidos descritores primários utilizando a lógica baseada em termos centrais. Os descritores foram trabalhados na língua inglesa vinculados por operadores booleanos (AND, OR e NOT), seguindo as regras descritas por Slamet et al. (2016) e vinculados ao tema proposto. Os descritores primários adotados farmi* OR agri* AND extens* AND entrepr* para artigos publicados $>2015<2021$.

O critério de inclusão e exclusão para esta rodada das recomendações aceitou apenas artigos completos. O intervalo de tempo utilizado na pesquisa foi definido de 2016 a 2021, ou seja, nos últimos 5 anos, sendo escritos na língua inglesa e que apresentam os descritores primários nos seguintes campos: título (title), resumo (abstract) e palavras-chave (keywords).

\section{Rodada 3: Seleção dos dados}

Pela possibilidade de se trabalhar com dois bancos de dados, a seleção inicial dos estudos foi realizada na sua primeira fase utilizando a "Busca por trabalhos duplicados" a partir da seleção dos artigos únicos com o emprego do software Start (State of the Art through Systematic Review).

Após a exclusão dos artigos duplicados, buscando atender os princípios das leis de Lotka e lei de Bradford, foi utilizado o método bibliométrico que inclui a categorização de 
acordo com a sumarização dos dados existentes nos artigos selecionados, e posterior extração de variáveis de interesse tais como: número de artigos publicados durante o período de 2015-2021, a distribuição de frequência da produtividade científica, principais clustering de origem (periódicos) pela Lei de Bradford e a rede colaborativa entre países considerando o mínimo de três citações entre eles.

O sistema StAt adota como principal classificador automático o método (SCAS) seleção automática de citação de pontuação. O SCAS, classifica os artigos utilizando duas fases, a primeira e dividida em dois recursos: por pontuação e por citação, já a segunda fase classifica em quadrantes (Octaviano, Silva e Fabbri, 2016).

Na primeira fase, o SCAS adota uma classificação de escore por valor em que, para cada ocorrência dos descritores no contidos nos campos: título, resumo e palavras-chave ele confere um determinado valor previamente atribuído. Nesta pesquisa foram consideradas as pontuações de ( 5 pontos no título, 3 pontos no resumo e 2 pontos nas palavras chaves, os descritores adotados nesta fase foram: Agriculture, Entrepreneurship, Extensionist, Farming e Tith.

Já na segunda fase do SCAS, o método posiciona os artigos duas categorias que contêm dois quadrantes cada hierarquicamente determinados pela Hipp (Hierarchical Point Placement). Paulovich \& Minghim (2008) descrevem as categorias da seguinte forma:

i. Categoria 1. São analisados e classificados automaticamente nesta categoria os artigos categorizados nos quadrantes Q1 e Q4 onde, o quadrante Q1 inclui automaticamente todos os artigos já analisados e aceitos, e o quadrante Q4 exclui automaticamente todos os artigos já analisados e excluídos;

ii. Categoria 2. Estão os artigos categorizados nos quadrantes Q2 e Q3 que são os estudos a serem revistos e reclassificados, estes são os artigos que apresentam pontuação elevada no segundo recursos da primeira fase do SCAS, porém não tem citações (Q3) ou apresentam ao menos uma citação (Q2), porém apresentam pontuação baixa. Nesta pesquisa por conveniência, foi adotada a premissa que, os artigos que classificados no quadrante Q2 que são os estudos com pontuação alta acima de 50\% do valor obtido pelo primeiro artigo de maior escore, mas sem citações estes foram revisados para determinar a sua relevância ou irrelevância para a pergunta da pesquisa, os demais classificados no quadrante já os que não atingirem esta pontuação assim como os que se localizaram nos quadrantes Q3 e Q4 foram rejeitados para seguir na próxima fase.

Rodada 4: Extração de estudos e dados 


\section{Extração dos estudos selecionados}

Selecionados os artigos mediante os procedimentos adotados na rodada anterior, foram desenvolvidos os critérios de Inclusão/Exclusão, descritos na Tabela 1 de modo a contemplar o início da seleção dos artigos classificados no quadrante 2 (Q2). Aqui, para que o artigo seja classificado para a próxima rodada ele deverá apresentar no mínimo dois critérios de Inclusão para poder ser elegível à próxima rodada.

Foram aceitos os artigos que apresentaram ao menos dois critérios para inclusão, estes trabalhos passaram para a fase de extração dos dados, em que foi realizada uma prospecção mais aprofundada sobre cada trabalho aceito, onde foram classificados os trabalhos que abordam especificamente a pergunta descrita na primeira rodada "A pesquisa científica que aborda, conjuntamente, o empreendedorismo e a produção agrícola familiar e campesina?".

Tabela 1 - Critérios de Inclusão/Exclusão dos artigos observados

\begin{tabular}{lc}
\hline \multicolumn{1}{c}{ Critérios } & Grupo de Indices \\
\hline Apresenta os dois descritores no título & Inclusão (I) \\
Periódico com Fator de impacto Internacional & Inclusão (I) \\
Correlação entre as duas áreas temáticas & Inclusão (I) \\
Automatically classified by SCAS & Inclusão ou Exclusão (I/E) \\
Não é trabalho sobre temas relacionados & Exclusão (E) \\
Não é trabalho sobre métodos inovadores & Exclusão (E) \\
Não apresenta solução inovadora & Exclusão (E) \\
$\begin{array}{l}\text { Não apresenta possibilidade } \\
\text { experimento }\end{array}$ & replicação do \\
$\begin{array}{l}\text { É uma revisão bibliográfica } \\
\text { Pouca aderência aos descritores primários }\end{array}$ & Exclusão (E) \\
\hline
\end{tabular}

Os demais trabalhos são considerados automaticamente inelegíveis para a próxima rodada. Todas as seleções nesta rodada foram realizadas por dois pesquisadores, de forma independente e restrita de modo a evitar influência pessoal nos resultados, as discrepâncias observadas durante esta rodada foram resolvidas por consenso. 


\section{Extração dos dados nos estudos selecionados}

Para a extração de dados dos trabalhos foram selecionados cinco descritores: Fator de impacto segundo Qualis capes 2016 para a área de avaliação Agricultura I pois, o CAPES classifica os estratos utilizando bases indexadoras tais como o JCR (Journal Citation Report) ou Scimago (2 years/doc). Cada estrato possui uma pontuação que varia de 0 a 100 , quando se classifica o Qualis utilizando o JCR podemos observar os valores de classificação como sendo: $\mathrm{A} 1>3.13, \mathrm{~A} 2=3.13, \mathrm{~B} 1=1.85, \mathrm{~B} 2=0.80, \mathrm{~B} 3=0.20$ e B4 $<0.20$; Classificação do modelo econômico agrícola (Agricultura familiar, Agricultura Campesina, Associação ou Cooperativismo); Sistema de produção (Agricultura ou Pecuária); Tipo de empreendedor segundo a definição de Zuini (2014) (Empreendedor Informal, Empreendedor Cooperado, Empreendedor Digital, Empreendedor Feminino, Empreendedor Individual, Empreendedor Social).

A seleção ocorreu manualmente de modo que fosse possível realizar uma leitura mais aprofundada, buscando a compreensão de todo o conteúdo desenvolvido nos manuscritos, assim como a relação de causa/efeito das atitudes empreendedoras relacionadas a produção agrícola em pequena escada tais como camponesa e agricultura familiar. Nesta fase foram excluídos os manuscritos que apresentavam relativa similaridades com os demais, assim como trabalhos que mantinham caráter de continuidade de uma pesquisa macro, pelos mesmos autores ou grupo de pesquisa.

Rodada 5: Análise e interpretação dos dados

$\mathrm{Na}$ análise e dos dados, buscando compreender a relação de citação de trabalhos e a participação dos autores das áreas de pesquisa relacionadas com o tema e atender os princípios das leis de produtividade de pesquisadores e de dispersão do conhecimento científico, nesta rodada foi adotado a análise redes de participação e citações baseadas em dados bibliométricos.

Para atender a fase de interpretação dos dados desta rodada, e buscando contemplar o princípio da lei de Zipf, foi utilizado como método análises lexicometrias e análises de discursos nos textos presentes com o auxílio do 'software' IRAMUTEQ (Marchand e Ratinaud, 2012). O 'software' utiliza Unidades de Contexto Iniciais (UCIs) para o desenvolvimento do modelo de análise proposto, nesta pesquisa foi utilizada a metodologia adotada por (SANTOS; HOLANDA; PEDROTTI; et al., 2020). Foram considerados como dados analíticos as Unidades de Contexto Iniciais (UCIs), Seguimentos de Texto (STs) e os 'cluster' resultantes da Categorização Hierárquica Descendente (CHD). 
Rodada 6: Síntese da revisão e qualificação dos trabalhos

Buscando atender os critérios de qualidade dos trabalhos selecionados, nesta fase de síntese, foram desenvolvidos códigos de indicadores de qualidade (Tabela 2), sendo adotado para a classificação por índice, o modelo de escala Likert partido do valor mais baixo 0 (Não atende o indicador) 0.5 (Atende parcialmente o indicador) para o valor mais alto 1 (Atende completamente o indicador). Os artigos que pontuaram com $>5.2$ foram considerados como muito alto, $5.2<2.6$ foram considerados alto, e artigos que alcançaram pontuação de $<2.5$ foram considerados baixos.

A categorização em ranking de prioridade os artigos baseados nos indicadores adotados para descrição de qualidade foram adotados três categorias: Muito alto ( 3 pontos), Alto (2 pontos), Baixo (1 ponto), tendo como base os dados categóricos do somatório dos indicadores de qualidade.

Tabela 2 - Indicadores adotados para descrição de qualidade dos artigos.

\begin{tabular}{clcc}
\hline Siglas & \multicolumn{1}{c}{ Indicador } & Grupo de Índice \\
\hline DME & Descreve o método/meios econômicos adotados & Índices de descrição \\
TEA & Apresenta o tipo de empreendedorismo adotado & Índices de descrição \\
ACR & Apresenta capacidade de reprodução & Índices de descrição \\
INV & Aparenta ser uma inovação & Índices de descrição \\
TEC & $\begin{array}{l}\text { Aplicação de uma tecnologia direcionada ao } \\
\text { empreendedorismo }\end{array}$ & Índices de descrição \\
DES & $\begin{array}{l}\text { Apresenta descrição detalhada do procedimento } \\
\text { metodológico }\end{array}$ & Índices de descrição \\
TAS & $\begin{array}{l}\text { Envolve tecnologia direcionada a área de agricultura } \\
\text { sustentável }\end{array}$ & Índices de descrição \\
LCD & Tem linguagem clara sobre os dados resultantes & Índices de descrição \\
\hline
\end{tabular}

A última rodada foi responsável pela classificação por qualidade dos trabalhos selecionados. Para identificar os artigos mais relevantes considerando as pontuações e classificações de toda a revisão, foi estabelecido um valor de corte correspondente a $85 \%$ do somatório dos critérios de classificação de escore por valor obtidos na rodada 3 (Seleção dos estudos). 
Os critérios de Inclusão/Exclusão obtidos na Rodada 4 (Extração de estudos e dados) atribuindo valores de 0 para não atendido e 2 para atendido, os valores de indicadores de qualidade obtidos na Rodada 6 (Síntese da revisão e qualificação dos trabalhos) em que foram atribuídos os valores de 0 para não atende o indicador, 0.5 atende parcialmente o indicador e 1 para atende o indicador assim como os valores alcançados no ranking de prioridade (3 Muito alto, 2 Alto e 1 Baixo).

O limite de $85 \%$ do somatório foi baseado no postulado de Pareto, que afirma que a maior parte do efeito se origina de um pequeno número de causas (Pareto, 1964). No contexto desta pesquisa, este postulado afirma que os artigos com maior pontuação representarão a maior parte do reconhecimento científico no conjunto bibliográfico atual (Azevedo, Ensslin e Jungles, 2014). Na Figura 1 é possível visualizar todo o processo da revisão sistemática adotada.

Figura 1 - Resumo do processo de revisão sistemática adotado

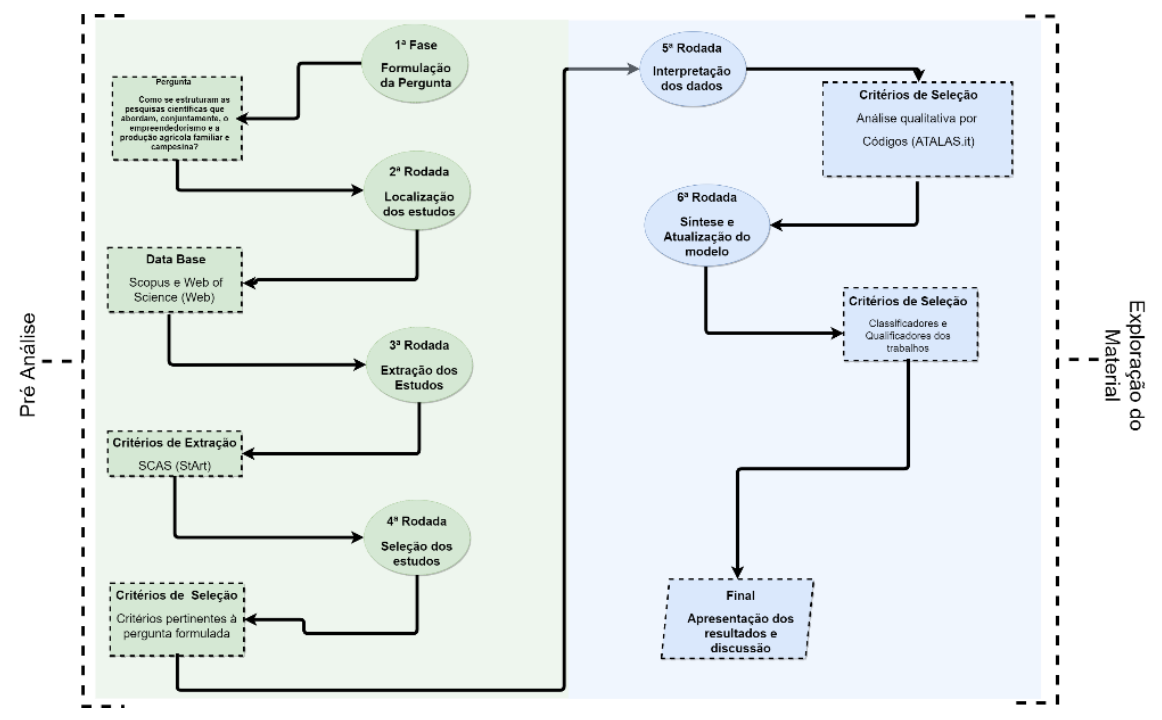

Fonte: Elaborado pelos autores, 2021.

Este método possibilitou compreender melhor a correlação entre os termos mais frequentes, as áreas de pesquisa assim como os objetivos de estudos mais frequentes.

\section{RESULTADOS E DISCUSSÃO}

Inicialmente a busca nas duas bases de dados resultaram em 400 artigos, 297 na Web of Science e 103 na Scopus, destes estudos encontrados, 23 foram considerados duplicados mediante a verificação de duplicidade e posteriormente excluídos. Todos os artigos não duplicados minerados nos bancos de dados (377 artigos) que emergiram utilizando os descritores de busca foram inicialmente considerados elegíveis para avançar para a rodada 3 que é a seleção dos estudos. 
Bibliometria dos artigos selecionados

Observando o acumulado de artigos publicados por autor, é possível notar que, a maior parte dos autores $60 \%$ contam com duas publicações, os demais contam com apenas uma publicação cada.

Na Figura 3 é possível observar os dez principais periódicos identificados no universo desta pesquisa. A maioria dos artigos foram publicados em três periódicos internacionais, sendo eles: Journal of Agricultural extension and rural development (31,6\%), Sustainability (11,6\%), Journal of global entrepreneurship research $(5,4 \%)$, os demais trabalhos foram publicados em diferentes periódicos, todos com apenas uma publicação cada, é possível observar também que, a maioria dos periódicos apresentam escopos especializados na área da extensão rural e sustentabilidade, demostrando o interesse e a relevância do tema focado para área rural.

Figura 3 - Dez principais periódicos presentes no corpus textual

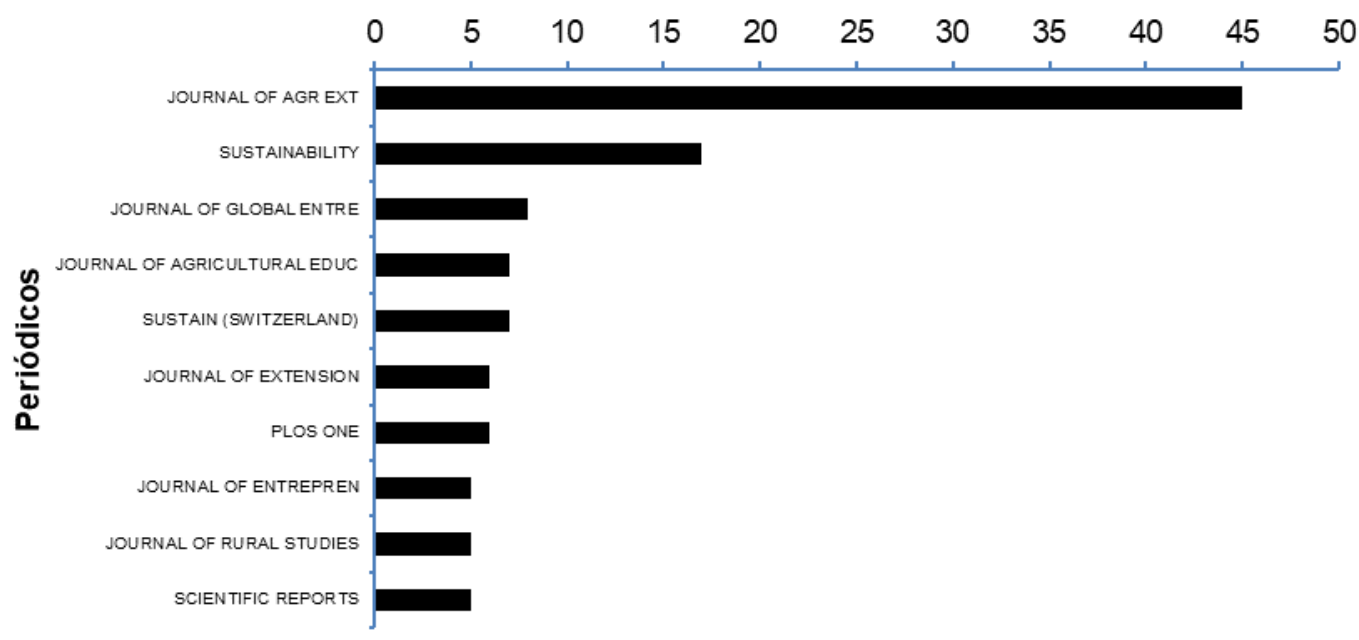

\section{Artigos publicados ( $\mathrm{n})$}

Fonte: Elaborado pelos autores, (2021).

Quando observado o número de citações de trabalhos por países (Figura 4), formou-se uma rede colaborativa de pesquisa que têm como principais países: Estados Unidos Reino Unido, China e Itália, onde o Estados Unidos e a China apresentam frequência 8 trocas de informações de pesquisa entre si, o reino Unido com a Itália 6 compartilhamentos.

Nesta mesma figura é possível observar que atualmente o Brasil realiza parcerias com diversos pesquisadores pelo mundo, tendo como seu maior parceiro a Alemanha com doze compartilhamentos e o Estados Unidos com sete trabalhos em parceria na área de empreendedorismo e agricultura extensiva. 
Figura 4 - Rede colaborativa entre países

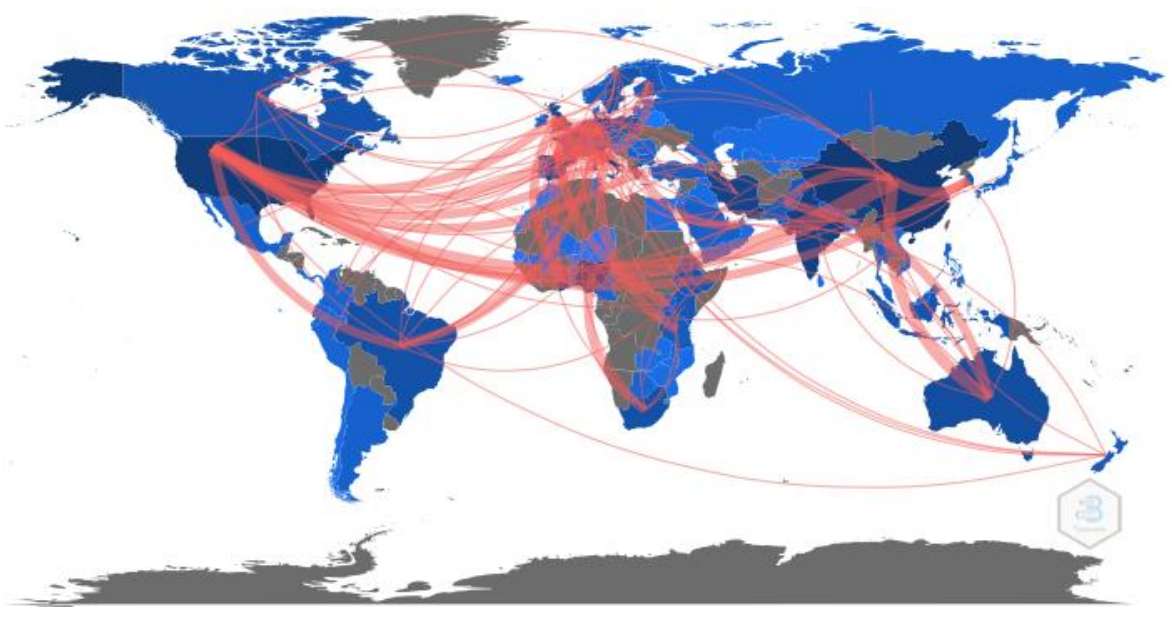

Fonte: Elaborado pelos autores, (2021).

\section{Seleção Automática de Citação de Pontuação}

O sistema SCAS categorizou automaticamente 122 artigos no quadrante Q4 e 14 artigos no quadrante Q3, sendo este último rejeitado para a próxima fase. O método categorizou 5 artigos no quadrante Q1 os quais foram aceitos automaticamente para a próxima rodada.

Os artigos classificados no Q2 (197 artigos), foram conduzidos para seleção manual buscando uma leitura mais aprofundada destes para posterior pontuação, destes 44 artigos alcançaram pontuação superior ao ponto de corte ( 22 pontos), 5 foram excluídos por não apresentar aderência entre os dois principais descritores analisados nesta pesquisa, e 2 por serem revisão sistemática. Os demais seguiram para as demais fases metodologias deste estudo, já que apresentavam pontuação significativamente relevante sobre a ótica de ocorrência dos descritores secundários no corpo textual.

\section{Resultados da extração dos estudos}

Os artigos contidos no quadrante Q1 abordam majoritariamente as mudanças da agricultura familiar e a inserção de novas tecnologias desenvolvidas para área de sistemas agrícolas classificados como pequenos produtores, a exemplo o trabalho de Moysiadis et al. (2021) que discute sobre o Smart Farming, este é um novo termo no setor agrícola que tem como o objetivo compreender a transformação das técnicas agrícolas adotadas na agricultura extensiva (técnicas básicas e tradicionais nas produções) em soluções inovadoras utilizando para isso a Tecnologia de Informação e Comunicação (TIC). 
Dos trabalhos que foram categorizados no Q1, o principal artigo atingiu 46 pontos pelo SCAS (Effect of virtual social networks on entrepreneurial behaviour ofagriculture students in Iran) (Farani et al., 2019) este trabalho aborda o comportamento empreendedor de estudantes da área das agrárias, o artigo destaca implicações para o desenvolvimento de novos planos pedagógicos buscando a formação de novos empreendedores, organizações de fomento e novos pesquisadores, destacando ainda o potencial das redes sociais virtuais para a promoção do empreendedorismo no meio acadêmico com futuro resultado para o campo.

Igualmente contidos neste quadrante estão pesquisas que apresentaram ter como objetivo, o papel do agricultor no sistema agrícola, tanto como receptor quanto como gerador ou codesenvolvedor de inovação (Phillips et al., 2019), explorando também o processo de mudança do campo e suas nuances, o estudo do empreendedorismo inovador no meio rural neste sentido tem implicações geográficas, econômicas, políticas e sociais para a comunidade envolvida (Jimenez et al., 2021; Phillips et al., 2019), tratando o empreendedorismo não como um fator singular, mas como um fator responsável pelo surgimento de ecossistemas de inovação e comunidades circulares destas inovações e negócios resultantes.

Síntese da revisão e qualificação dos trabalhos

\section{Análise qualitativa dos dados textuais}

Buscando analisar todos os conjuntos de textos (artigos) selecionados, a preparação inicial dos corpus textuais se deu com a criação em um único corpus contendo todos os resumos, títulos e palavras-chaves dos artigos selecionados na fase de extração dos dados. Desta forma, dos 44 artigos selecionados considerados aqui UCIs, organizados em um único arquivo.

As 44 UCIs retornaram uma $f=269$ STs, nos quais continham 9826 ocorrências (Vocábulos, Palavras e Formas) destas, 1729 palavras distintas e 768 hapax (palavras com uma única ocorrência). Exibindo uma frequência de 7,82\% para ocorrência de palavras, e uma frequência média de 44,42\% de ocorrência por STs. Após seleção dos vocábulos as suas raízes válidas surgiram 207 STs, representando uma taxa de retenção de 76,95\% do corpus, satisfazendo os critérios sugeridos pela literatura pertinente.

A partir da geração do Diagrama de Zipf (Figura 5), observando eixo frequences (y) que apresenta as frequências das palavras por ordem decrescente, e o eixo rangs (x) que apresenta a quantidade das palavras, ambos em escalas logarítmicas (Lebart e Salem, 1988), é possível inferir que, um elevado número de palavras se repetiu poucas vezes (nesse caso o intervalo acima de 500 palavras) e poucas palavras que se repetem muitas vezes (uma mesma forma 
repetida aproximadamente 500 vezes), resposta observada também por Leite et al. (2017) utilizando o Iramuteq.

Figura 5 - Diagrama de Zipf do corpus.

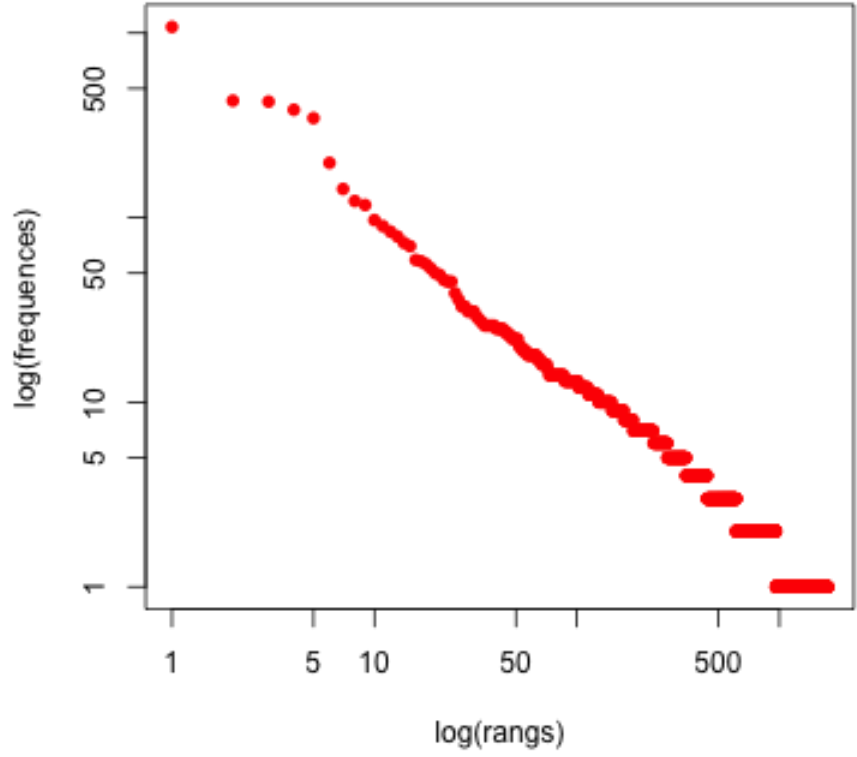

Fonte: Elaborado pelos autores, 2021.

Os STs válidos resultaram em quatro classes organizadas segundo o teste estatístico quiquadrado $\left(\chi^{2}\right)$, teste este que demonstra a força associativa entre as palavras contidas em cada classe (Camargo e Justo, 2013). Ele considera como boa força associativa o $\left(\chi^{2}\right)$ apresentando valores acima de 3,84, representando p-value <0,0001 (SOUZA, et al., 2018). Na Figura 6, é possível observar o dendrograma representativo das Classes (clusters) obtidos pela Classificação Hierárquica Descendente (CHD) formada pelo corpus textual analisado, neste sentido foi possível alcançar o pressuposto da lei de Zipf voltado a verificar os principais termos e agrupamentos formados. 
Figura 6 - Dendrograma representativo das Classes6 observadas nos artigos de pesquisa.

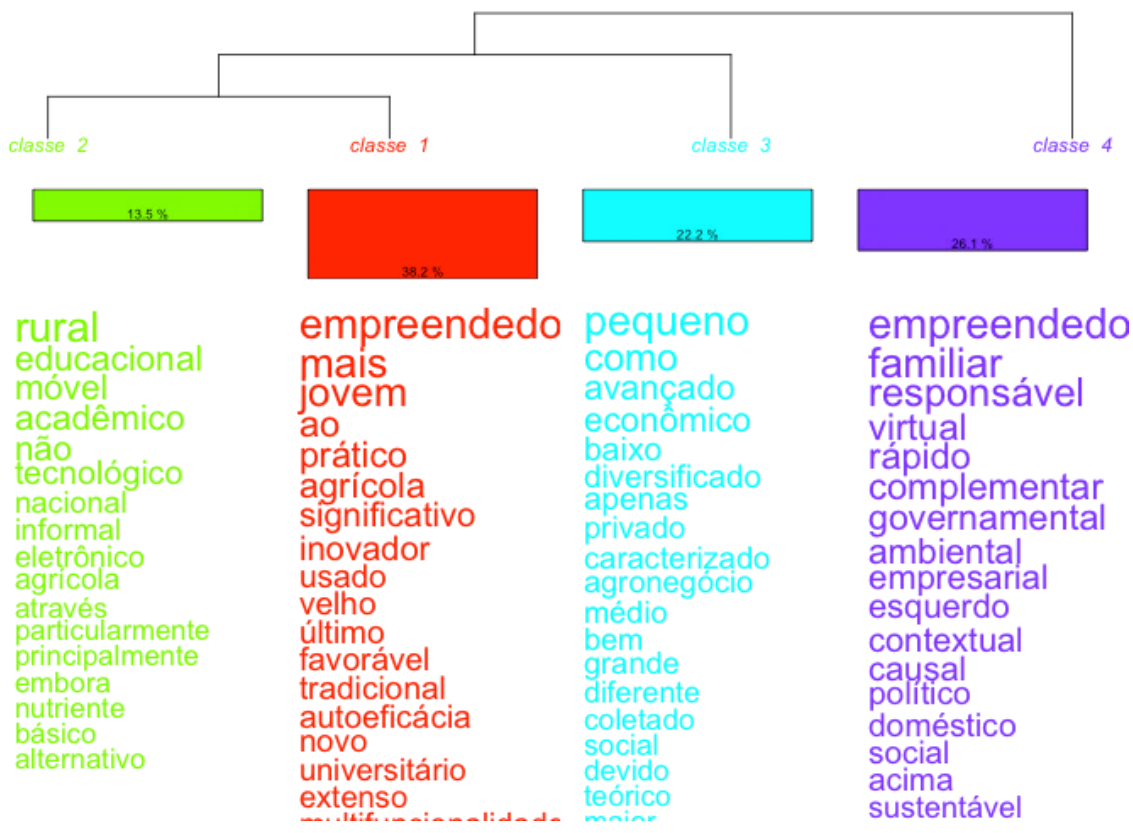

Fonte: Elaborado pelos autores, 2021.

A classe 1 (Empreendedorismo Digital) trata empreendedorismo Digital e as suas vertentes para a agricultura, esta classe agrupou $38,16 \%(f=79 / 207 \mathrm{STs})$ válidos. O seguinte segmento de texto observado descreve o conteúdo da classe: "O objetivo desta investigação é avaliar o impacto do programa de carteira eletrônica do Governo Federal da Nigéria no desenvolvimento do empreendedorismo agrícola informal na Nigéria rural" (Uduji, OkoloObasi e Asongu, 2019), a classe também aborda sobre e a adoção de tecnologias quem lidam com a agricultura de precisão e o meio digital, assim como o espectro psicológico das percepções quanto a aceitação delas pelo produtor extensivo (Andrade et al., 2020; Vecchio et al., 2020).

Este tema em questão traz consigo uma grande relevância, visto que, a nova produção extensiva precisa atualmente configurar-se como uma atividade que seja rentável, proporcione qualidade de vida para os produtores e ao mesmo tempo não ocasione impactos ambientais excessivos (Naharro, 2019).

A classe 2 (Educação em empreendedorismo) apresenta 13.73\% dos STs válidos. Esta classe aborda textos relacionados a metodologias pedagógicas e projetos educacionais tais como de

${ }^{6}$ Classes com grau de significância estatística com a classe de $<0,0001$ 
extensão rural e inovação. O seguimento de texto "Programa educacional e de integração acadêmica e tecnológica PEIAT para jovens fora da escola em conflitos e áreas rurais" (Saliot e Tabinas, 2017) descreve melhor a classe observada.

As duas classes emergem do mesmo agrupamento, comportamento que demonstrando, uma estreita relação dos textos que tratam de educação empreendedora e a inovação representada aqui com as novas tecnologias direcionadas a agricultura, esse somatório vai ao encontro da perspectiva do ensino ao empreendedorismo para o desenvolvimento da inovação, tema bastante abordado em novas pesquisas acadêmicas sobre educação, Além disso, nesta classe são apresentados trabalhos que fornecessem estratégias de ação necessárias para desenvolver um empreendedorismo responsável e voltado para a campo (SANTOS et al., 2019; SILVA et al., 2017).

Nas últimas duas décadas, tem havido uma valorização crescente de que a trajetória de carreira empreendedora tem um papel vital a desempenhar na mudança social em direção à carreira (Mohammadinezhad e Sharifzadeh, 2017), principalmente ao profissional do campo, desta forma, cresce em conjunto a quantidade de estudos relacionados com o tema, provável fator de surgimento desta classe com um número maior de artigos.

A classe 3 (Empreendimentos agrícolas) surge de um agrupamento superior, vinculado ao agrupamento referente as classes 1 e 2 . A classe 3 corresponde a 22,22\% dos seguimentos analisados ( $f=46$ STs), nela estão contidos os seguimentos que abordam a diversidade dos empreendimentos agrícolas pertencentes ao sistema extensivo estudado, o artigo escrito por Khapayi et al. (2018) descreve melhor a classe "Esta pesquisa investigou a eficácia da agricultura por contrato sem comercialização de pequenos produtores de hortaliças". Tais artigos corroboram com a ligação do agrupamento com os anteriores analisados, onde é vinculada a tecnologia digital e estudos acadêmicos em diferentes categorias de empreendimentos agrícolas.

Os artigos selecionados nesta classe trabalham também novos paradigmas na literatura acerca do perfil do atual trabalho no campo e pesquisas relacionadas a extensão rural, como, por exemplo a noção de empreendedores agrícolas multifamiliares, em detrimento ao termo agricultura familiar relacionando apenas fator família (Jimenez et al., 2021; Saliot e Tabinas, 2017; Sippel, 2016).

Os estudos de caráter sociológicos da interação trabalho no campo e a relação familiar se mostra importante a medida que, o empreendimento agrícola familiar atravessa por mudanças significativas em seu núcleo laboral, já que, atualmente os descendentes procuram novos meios 
de trabalho longe do campo reduzindo a mão de obra e forção os proprietários a contratarem mão de obra (Loper Aires e Salamoni, 2012).

E por fim a classe 4 (Intensão empreendedora) mostrou ser a classe mais afastada das demais que estão no segundo agrupamento (a maior classe), dela surgem artigos que tratam sobre os contextos psicológicos do empreendedorismo no campo, tais como: intensão empreendedora, percepção dos modelos aplicados, relacionamento interpessoal e contextos de associativismo e cooperativismo. O seguimento de texto exemplifica esta classe "Os fatores que inibem o empreendedorismo no contexto da agricultura familiar incluem a insegurança da posse da terra [...] e o afastamento dos sistemas de irrigação", (Wale, Unity e Nolwazi, 2021).

Analisando a Análise Fatorial de Correspondência que identifica a proximidade entre as classes observadas e suas formas (palavras) (Figura 7), é possível observar que os termos "Empreendedorismo" (X2 > 29,32) e "Rural" (X2 53,2) são termos centrais do AFC demostrando que o, os artigos são relevantes como base para a resposta da questão adotada nesta pesquisa (como se estruturam as pesquisas científicas que abordam, conjuntamente, o empreendedorismo e a produção agrícola extensiva?).

Figura 7 - Análise Fatorial de Correspondência com base nas categorias

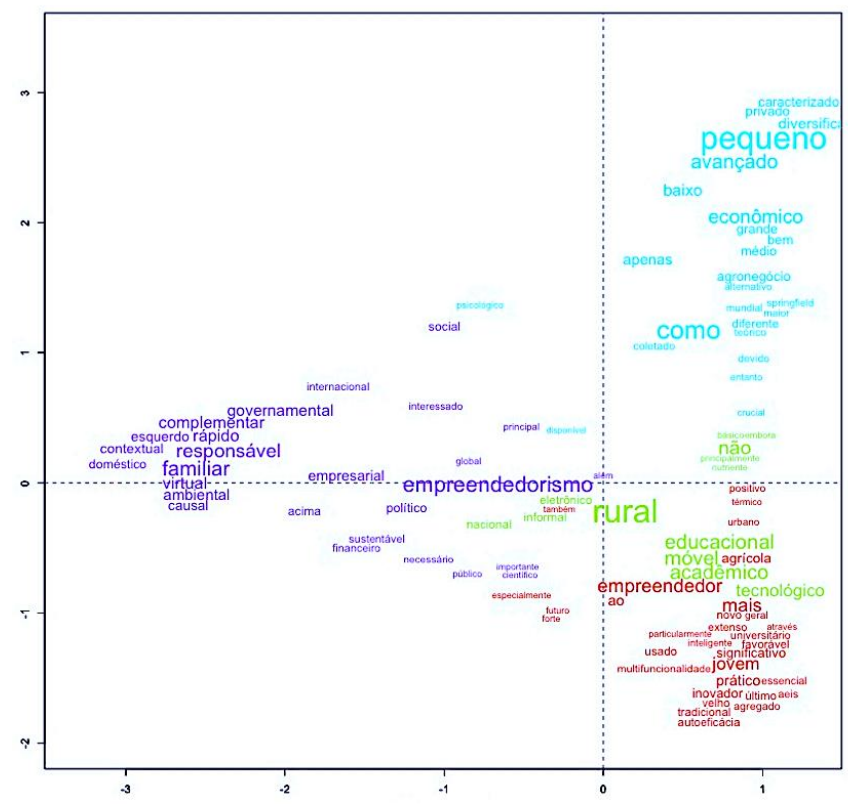

Fonte: Elaborado pelos autores, 2021. 
As palavras que foram posicionadas mais distantes demonstram que foram pouco ou não utilizadas no mesmo contexto das mais centrais, a exemplo deste comportamento temos o termo "Pequeno", esta palavra está contida na Classe 3 representando um X2 $>52,55$ na classe, valor relevante para uma frequência de palavras, porém está posicionada em um quadrante mais distante das demais classes (Figura 5), isso ocorre por uma baixa representação de trabalhos no corpus textual que estudam as intenções empreendedoras presentes nos pequenos produtores.

Síntese da qualidade dos estudos selecionados

A partir das análises apresentadas anteriormente, partiu-se para uma análise qualitativa mais minuciosa dos resumos selecionados, sendo possível a classificação dos artigos por meio do somatório dos valores obtidos em todas as rodadas e realizando o corte correspondente a $85 \%$ do somatório de todos os critérios foi possível chegar a sete artigos que expressam bem o perfil dos trabalhos que abordam o empreendedorismo direcionado a agricultura extensiva (Tabela 3).

É importante observar que, artigos selecionados pelo método corroboram com os achados no método anterior de alocação por Classificação Hierárquica Descendente (CHD), corroborando assim outros métodos que utilizando o princípio de Pareto como base para seleção dos trabalhos (Carvalho et al., 2020; Costa-Carrapiço, Raslan e González, 2020), dado que, segundo este princípio, os artigos que compõem 20\% dos achados podem expressar bem o corpus textual estudado.

Tabela 3 - Artigos com maior pontuação após somatório da síntese de qualidade

\section{CHD com \\ maior \\ ocorrência}

Título do artigo

Ano de

publicação

Wale, Z. E., Unity, C., \& Nolwazi, H. (2021). Towards identifying enablers and

3 e 4 inhibitors to on-farm entrepreneurship: evidence from smallholders in

KwaZulu-Natal, South Africa. Heliyon, 7(1).

Gholamrezai, S., Aliabadi, V., \& Ataei, P. (2021). Recognizing dimensions of sustainability entrepreneurship among local producers of agricultural inputs. Journal of Environmental Planning and Management. doi: $10.1080 / 09640568.2021 .1875998$

Mohammadinezhad, S., \& Sharifzadeh, M. (2017). Agricultural

4 entrepreneurship orientation: is academic training a missing link? Education and Training, 59(7-8), 856-870. doi: 10.1108/ET-10-2016-0156

Ifeanyi-obi, C. C., \& Ewurum, T. (2019). Influence of Entrepreneurship

1 e 4 Education on Career Aspiration of Agriculture Undergraduates in Rivers State.

Journal of Agricultural Extension, 23(4), 107-118. doi: 10.4314/jae.v23i4.13 
Yoshida, S., Yagi, H., Kiminami, A., \& Garrod, G. (2019). Farm diversification and sustainability of multifunctional peri-urban agriculture: Entrepreneurial attributes of advanced diversification in Japan. Sustainability (Switzerland), 11(10). doi: 10.3390/su11102887

Shahraki, H., \& Heydari, E. (2019). Rethinking rural entrepreneurship in the

2 era of globalization: some observations from Iran. Journal of Global Entrepreneurship Research, 9(1). doi: 10.1186/s40497-019-0162-6

Far, S. T., \& Rezaei-Moghaddam, K. (2019). Multifunctional agriculture: an

1 e 2 approach for entrepreneurship development of agricultural sector. Journal Of Global Entrepreneurship Research, 9(1). doi: 10.1186/s40497-019-0148-4

Ainda a partir da análise da Tabela 3, observa-se a correspondência da quantidade de artigos selecionados com a análise realizada no CHD apresentado na Figura 7, visto que fica evidente a existência dos "clusters" majoritários. (1 e 4) voltados a representar os artigos que discutem o "Empreendedorismo Digital"; e o outro que representa os estudos sobre os "Intenção empreendedora", assim como as proximidades entre estes e os demais "clusters".

\section{CONSIDERAÇÕES FINAIS}

O principal fio condutor desta pesquisa foi averiguar quais os últimos avanços do empreendedorismo agrícola para o meio agrícola com atividade extensiva através de uma análise sistemática da literatura internacional. Partido deste questionamento, pode-se perceber que grande parte das publicações relacionadas ao empreendedorismo de empresas agrícolas extensivas envolvem empreendedorismo digital (categoria 1) e adoção de tecnologias de agricultura de precisão, que estão diretamente relacionadas ao modelo de inovação de sistemas agrícolas e agricultura digital.

A partir da seleção do artigo principal e dos seus dados observacionais, devido ao campo do conhecimento, observações separadas têm aparecido repetidamente na pesquisa empírica, mas são raramente estudadas em simultâneo.

Pode-se inferir que o método SCAS utilizando nesta pesquisa satisfaz o objetivo proposto de satisfazer a lei da dispersão do conhecimento científico ou a lei de Bradford e a lei da distribuição e frequência de palavras ou lei de Zipf, pois usando este método, o trabalho principal pode ser selecionado por meio das palavras e Citações, respondendo assim às questões utilizadas nesta pesquisa.

Utilizando o programa IRAMUTEQ, buscando considerar os dados relativos à aparência do texto, atendendo ao princípio da lei de Zipf traz as vantagens de categorizar e organizar o texto, ao mesmo tempo que sacrifica as quatro categorias que aparecem, para que 
seja possível agrupar e relacionar-se com o texto desta forma, uma definição mais precisa de núcleo refere-se ao tópico mais relevante.

Quanto aos resultados observados nas rodadas de qualidade, nota-se que esses resultados se confirmam com os segmentos de texto selecionados pelo método de classificação hierárquica decrescente, resultados que podem ser explicados pelo princípio de Pareto, em que, para muitos eventos, cerca de $80 \%$ Resultado é $20 \%$ da causa.

Ressalta-se que a utilização de duas bases de dados (Scopus e Web of Science) pode alinhar diversos trabalhos emergentes de diferentes periódicos e até campos do conhecimento científico, o que permite que a amostra inclua mais pesquisas, mas isso significa que o número de trabalhos sem sentido a aparência, assim como o grande número de obras incluídas no quadrante Q4 e a exclusão inicial automática, indicam que esses temas não estão totalmente estabelecidos e consolidados na coleção, e indicam que aparecem as áreas centrais e dispersas de um tema no mesmo texto Com base nessas ideias, em muitos casos, esse fator está relacionado à pesquisa individual ou ao surgimento de pequenos grupos locais.

\section{REFERÊNCIAS}

ANDRADE, C. E. C. DE; HOLANDA, F. S. R.; UBIRAJARA, W. DE M.; BANDEIRA, A. A.; SANTOS, L. D. V. Uma análise bibliométrica da literatura aplicada a transferência de tecnologia em células a combustível. Research, Society and Development, v. 9, n. 12, p. e22391211021-e22391211021, 28 dez. 2020.

ARIA, M.; CUCCURULLO, C. bibliometrix: An R-tool for comprehensive science mapping analysis. Journal of informetrics, v. 11, n. 4, p. 959-975, 2017.

AZEVEDO, R. C. DE; ENSSLIN, L.; JUNGLES, A. E. A Review of Risk Management in Construction: Opportunities for Improvement. Modern Economy, v. 05, n. 04, p. 367-383, 3 abr. 2014.

BELL, R. F.; DAHL, J. B.; MOORE, R. A.; KALSO, E. Peri-operative ketamine for acute postoperative pain: a quantitative and qualitative systematic review (Cochrane review). Acta Anaesthesiologica Scandinavica, v. 49, n. 10, p. 1405-1428, 19 nov. 2005.

CAMARGO, B. V.; JUSTO, A. M. IRAMUTEQ: um software gratuito para análise de dados textuais. Temas em psicologia, v. 21, n. 2, p. 513-518, 2013.

CARVALHO, G. D. G. DE; SOKULSKI, C. C.; SILVA, W. V. DA; CARVALHO, H. G. DE; 
MOURA, R. V. DE; FRANCISCO, A. C. DE; VEIGA, C. P. DA. Bibliometrics and systematic reviews: A comparison between the Proknow-C and the Methodi Ordinatio. Journal of Informetrics, v. 14, n. 3, p. 101043, 1 ago. 2020.

CASSETTARI, R.-R.-B.; PINTO, A.-L.; RODRIGUES, R.-S.; SANTOS, L.-S. Comparação da Lei de Zipf em conteúdos textuais e discursos orais. Profesional de la Información, v. 24, n. 2 SE-Artículos de investigación / Research articles, 11 mar. 2015.

CHANDLER, J.; CUMPSTON, M.; THOMAS, J.; HIGGINS, J. P.; DEEKS, J. J.; CLARKE, M. J. Cochrane Capítulo I: Introdução | Treinamento. Disponível em: <https://training.cochrane.org/handbook/current/chapter-i>. Acesso em: 20 ago. 2021. COSTA-CARRAPIÇO, I.; RASLAN, R.; GONZÁLEZ, J. N. A systematic review of genetic algorithm-based multi-objective optimisation for building retrofitting strategies towards energy efficiencyEnergy and BuildingsElsevier, , 1 mar. 2020.

CUÉLLAR-GÁLVEZ, D.; ARANDA CAMACHO, Y.; MOSQUERA-VÁSQUEZ, T. A Model to Promote Sustainable Social Change Based on the Scaling up of a High-Impact Technical Innovation. Sustainability, v. 10, p. 4532, 1 dez. 2018.

FARANI, A. Y.; KARIMI, S.; IZADI, N.; ATAEI, P. Effect of virtual social networks on entrepreneurial behaviour of agriculture students in Iran. Applied economics, v. 51, n. 21, p. 2326-2335, 2019.

HERNANDES, E.; ZAMBONI, A.; FABBRI, S.; THOMMAZO, A. DI. Using GQM and TAM to evaluate StArt - a tool that supports Systematic Review. CLEI Electronic Journal, v. 15, p. 3,2012 .

IDRI, A.; ABNANE, I.; ABRAN, A. Evaluating Pred (p) and standardized accuracy criteria in software development effort estimation. Journal of Software: Evolution and Process, v. 30, n. 4, p. e1925, 2018.

JIMENEZ, I. A. C.; GARCÍA, L. C. C.; VIOLANTE, M. G.; MARCOLIN, F.; VEZZETTI, E. Commonly used external tam variables in e-learning, agriculture and virtual reality applications. Future Internet, v. 13, n. 1, p. 1-21, 2021.

JULIEN, P. A. Empreendedorismo regional e economia do conhecimento. Saraiva Educação SA, 2017.

KEELE, S. Guidelines for performing systematic literature reviews in software engineering. Technical report, Ver. 2.3 EBSE Technical Report. EBSE, 2007. 
KHAPAYI, M.; NIEKERK, P. VAN; CELLIERS, P. R. Challenges of Contract Farming among Small-Scale Commercial Vegetable Farmers in Eastern Cape South Africa. Journal of agricultural extension, v. 22, n. 3, p. 195-206, out. 2018.

LEBART, L.; SALEM, A. Analyse statistique des données textuelles: questions ouvertes et lexicométrie. Dunod Paris, 1988.

LEITE, P.; SOUZA, E.; SILVA, A.; LEITE, D. O ambiente de gestão de MPEs do setor de turismo no Brasil: uma revisão sistemática The management environment at MPEs in the tourism sector at Brazil: a systematic review. 11 fev. 2017.

LOPER AIRES, C. H.; SALAMONI, G. Agricultura familiar e as relações sociais de trabalho:um estudo sobre a pluriatividade na Vila Freire - Cerrito - RS. Geografia Ensino \& Pesquisa, v. 17, n. 1, p. 41-54, 22 nov. 2012.

MARCHAND, P.; RATINAUD, P. L'analyse de similitude appliquée aux corpus textuels: les primaires socialistes pour l'élection présidentielle française. Actes des 11eme Journées internationales d'Analyse statistique des Données Textuelles. JADT, v. 2012, p. 687-699, 2012.

MEDEIROS, I. L. DE; VIEIRA, A.; BRAVIANO, G.; GONÇALVES, B. S. Revisão Sistemática e Bibliometria facilitadas por um Canvas para visualização de informação. InfoDesign - Revista Brasileira de Design da Informação, v. 12, n. 1, p. 93-110, 15 out. 2015 .

MOHAMMADINEZHAD, S.; SHARIFZADEH, M. Agricultural entrepreneurship orientation: is academic training a missing link? Education and training, v. 59, n. 7-8, p. 856-870, 2017.

MOYSIADIS, V.; SARIGIANNIDIS, P.; VITSAS, V.; KHELIFI, A. Smart Farming in Europe. Computer science review, v. 39, 2021.

NAHARRO, N. Agricultura Familiar. In: La pobreza en la prensa. [s.l: s.n.]. v. 5p. 57-62. OCTAVIANO, F.; SILVA, C.; FABBRI, S. Using the SCAS strategy to perform the initial selection of studies in systematic reviews: an experimental study: EASE '16.Association for Computing Machinery, 28 out. 2016Disponível em:

<https://doi.org/10.1145/2915970.2916000>

PARETO, V. Cours d'économie politique. Librairie Droz, 1964. v. 1

PAULOVICH, F. V; MINGHIM, R. Hipp: A novel hierarchical point placement strategy and its application to the exploration of document collections. IEEE Transactions on Visualization 
and Computer Graphics, v. 14, n. 6, p. 1229-1236, 2008.

PHILLIPS, P. W. B.; RELF-ECKSTEIN, J.-A. A.; JOBE, G.; WIXTED, B. Configuring the new digital landscape in western Canadian agriculture. NJAS - Wageningen Journal of Life Sciences, v. 90-91, 2019.

RIEGLER, M. Insect threats to food security. Science, v. 361, n. 6405, p. 846-846, ago. 2018. ROCKSTROM, J.; WILLIAMS, J.; DAILY, G.; NOBLE, A.; MATTHEWS, N.; GORDON, L.; WETTERSTRAND, H.; DECLERCK, F.; SHAH, M.; STEDUTO, P. Sustainable intensification of agriculture for human prosperity and global sustainability. Ambio, v. 46, n. 1, p. 4-17, 2017.

SALIOT, A.; TABINAS, R. J. Academic and technology integration-educational program (ATIEP) for out-of-school-youths (OSY) in conflict and rural areas of the Philippines. Rural extension and innovation systems journal, v. 13, n. 2, p. 151-155, 2017.

SALVIATI, M. E. Manual do Aplicativo IramuteqIamuteq, , 2017. Disponível em: $<$ http://www.iramuteq.org/documentation/fichiers/manual-do-aplicativo-iramuteq-par-mariaelisabeth-salviati>

SANTANA, A. P. S. DE. A diversificação de cultivos na sustentabilidade da agricultura familiar no município de Lagarto-SE. Universidade Federal de Sergipe, 18 dez. 2014. SANTOS, L. D. V. et al. Empreenda AGRO Sustentável. Empreenda AGRO Sustentável, 3 fev. 2019.

SANTOS, L. D. V.; HOLANDA, F. S. R.; PEDROTTI, A.; ANDRADE, C. E. C. DE; BANDEIRA, A. A. Prospecção tecnológica sobre PD\&I em Recursos Hídricos. Revista IberoAmeriana de Ciências Ambientais, p. 25, 2020.

SILVA, F. DA C. E; MANCEBO, R. C.; MARIANO, S. R. H. Educação empreendedora como método: o caso do minor em empreendedorismo inovação da UFF. Revista de Empreendedorismo e Gestão de Pequenas Empresas, v. 6, n. 1 (Janeiro/Abril), p. 196-216, 11 nov. 2017.

SIPPEL, S. R. Breaking ground: Multi-family farm entrepreneurs in Moroccan export agriculture. Journal of Rural Studies, v. 45, p. 279-291, 2016.

SLAMET, C.; RAHMAN, A.; RAMDHANI, M. A.; DARMALAKSANA, W. Clustering the verses of the Holy Qur'an using K-means algorithm. Asian Journal of Information Technology, v. 15, n. 24, p. 5159-5162, 2016. 
SOUZA, M. A. R. DE; WALL, M. L.; THULER, A. C. DE M. C.; LOWEN, I. M. V.; PERES, A. M. O uso do software IRAMUTEQ na análise de dados em pesquisas qualitativas. Revista da Escola de Enfermagem da USP, v. 52, n. 0, 19 mar. 2018.

UDUJI, J. I.; OKOLO-OBASI, E. N.; ASONGU, S. A. The impact of e-wallet on informal farm entrepreneurship development in rural Nigeria. Electronic Journal of Information Systems in Developing Countries, v. 85, n. 3, 2019.

VECCHIO, Y.; ROSA, M. DE; ADINOLFI, F.; BARTOLI, L.; MASI, M. Adoption of precision farming tools: A context-related analysis. Land use policy, v. 94, maio 2020.

WALE, Z. E.; UNITY, C.; NOLWAZI, H. Towards identifying enablers and inhibitors to onfarm entrepreneurship: evidence from smallholders in KwaZulu-Natal, South Africa. Heliyon, v. 7, n. 1, jan. 2021.

XIAO, D.; TAN, Y.; LIU, X.; YANG, R.; ZHANG, W.; HE, X.; WANG, K. Effects of different legume species and densities on arbuscular mycorrhizal fungal communities in a karst grassland ecosystem. Science of the Total Environment, v. 678, p. 551-558, 2019.

ZUINI, P. Os 9 tipos de empreendedores mais comuns no Brasil. Disponível em: $<$ https://exame.com/pme/os-9-tipos-de-empreendedores-mais-comuns-no-brasil/>. Acesso em: 10 jul. 2021.

\section{Recebido em: 01/06/2021}

Aprovado em: 20/06/2021

Publicado em: 30/06/2021 